\title{
Characteristics of presystolic flow in the superior vena cava: new thoughts on a forgotten sound
}

\author{
S J D Brecker, H B Xiao, M Mbaissouroum, D G Gibson
}

\begin{abstract}
Cross sectional, $M$ mode, and Doppler echocardiography, apexcardiography, and phonocardiography were used to characterise presystolic cardiovascular sounds in three patients with ventricular disease. Although the aetiology was different (dilated cardiomyopathy, primary pulmonary hypertension, and chronic pulmonary thromboembolic disease), in each case the presystolic sound was associated with a rapid change in acceleration of blood and with flow reversal in the superior vena cava, and could only be recorded at the right sternal edge or over the jugular veins. Such flow characteristics may be explained by a raised ventricular end diastolic pressure with reduced compliance. Use of these techniques helps to understand the cause of a previously described but little recognised heart sound, and adds weight to the interpretation of its presence in disease.
\end{abstract}

Heart sounds may be classified into those originating from the valves, which coincide with the final halt of opening and closing of the valves and are of high frequency, and added ventricular filling sounds, which are of low frequency and comprise the third sound, the atrial or fourth sound, or summation of the two. ${ }^{1}$ Such added heart sounds occurring during diastole are important physical findings and often indicate ventricular disease. With non-invasive techniques such as Doppler, phono, and echocardiography, we can study the direction, position, and velocity of blood flow within the heart at the instant an added sound occurs. Such techniques have shown that the onset of a third sound occurs at the time of peak transmitral flow velocity (during rapid filling), whereas a fourth sound usually occurs at the beginning of flow at the start of atrial systole. ${ }^{2}$ The mechanisms are thus different from those responsible for the first and second sounds. We now report three cases in which presystolic sounds were identified by phonocardiography. The aetiology in each case was different, yet similar pathophysiological mechanisms have been identified. This added sound has features different from diastolic sounds usually present in patients with ventricular disease, so we analysed the mechanisms responsible.

\section{Methods}

Cross sectional and $M$ mode echocardiograms were taken with the patient in the standard left lateral position with an Advanced Technical Laboratory 860C Imager with a 3.5 $\mathrm{MHz}$ mechanical transducer. We recorded Doppler signals with a Doptek Spectrascan and a $2 \mathrm{MHz}$ transducer. Peak transmitral and transtricuspid flow velocities were identified by continuous wave and were recorded in pulsed wave mode with a $3 \mathrm{~mm}$ gate and $250 \mathrm{~Hz}$ wall filter. Regurgitant flow was identified and recorded in continuous mode. Apexcardiograms were recorded from the point of maximal impulse by a Cambridge Instruments transducer with a time constant of four seconds. Phonocardiograms were recorded from a Leatham microphone with a low frequency filter. Apexcardiograms, $M$ mode echocardiograms, and Doppler traces were recorded separately with simultaneous electrocardiogram and phonocardiogram on a Honeywell (Ecoline 22) strip chart recorder at a paper speed of $10 \mathrm{~cm} / \mathrm{s}$.

\section{Case reports}

\section{PATIENT 1}

A 46 year old woman with previous good health presented with chest pain and breathlessness. Physical examination revealed tachycardia, raised venous pressure $(20 \mathrm{~cm}$ above the sternal angle), ankle oedema, and cardiomegaly. Her chest $x$ ray film showed pulmonary oedema, although her electrocardiogram and cardiac enzyme concentrations were normal. A diagnosis of viral myopericarditis was made during a prolonged hospital admission, and she was discharged on diuretics and angiotensin converting enzyme inhibitors. She was readmitted four months later with increasingly severe dyspnoea, orthopnoea, and ankle swelling. Pansystolic murmurs were audible at the apex and lower left sternal edge. Chest $x$ ray film showed an enlarged cardiac shadow with pulmonary venous congestion, and her electrocardiogram remained unchanged from the previous recording with a heart rate of 120 and PR interval of $0.16 \mathrm{~s}$. She was referred for echocardiographic assessment. This showed biventricular enlargement with global hypokinesis. No convincing added sound could be heard with phonocardiography from the left sternal edge. An unimpressive low frequency deflection was present at the peak of rapid filling, but after the start of the succeeding $P$ wave (fig

\author{
Royal Brompton Nation \\ Heart and Lung Hospital \\ Sydney Street, Chelsea, \\ London SW3 6NP. \\ Accepted for publication \\ 9 February 1992 \\ Cardiac Departm \\ Lung Hospital, London \\ H B Xiao \\ D G Gibson
}


Figure 1 Pulsed wave Doppler recordings of from patient 1, with simultaneous

electrocardiogram (ECG and phonocardiogram $100 \mathrm{~ms}$ are marked. (A) Phonocardiogram recorded from lower left sternal edge. The only possible diastolic deflection recorded from this site coincides with the peak forward transmitral flow, characteristic of a summation sound.

(B) Jugular venous loud presystolic sound $(S)$, 140 ms after the $P$ wave occurring after peak

forward flow when the velocity is decreasing. Full scale deflection is $4 \mathrm{kHz}$. transmitral flow velocity (PCG). Time intervals of phonocardiogram shows a
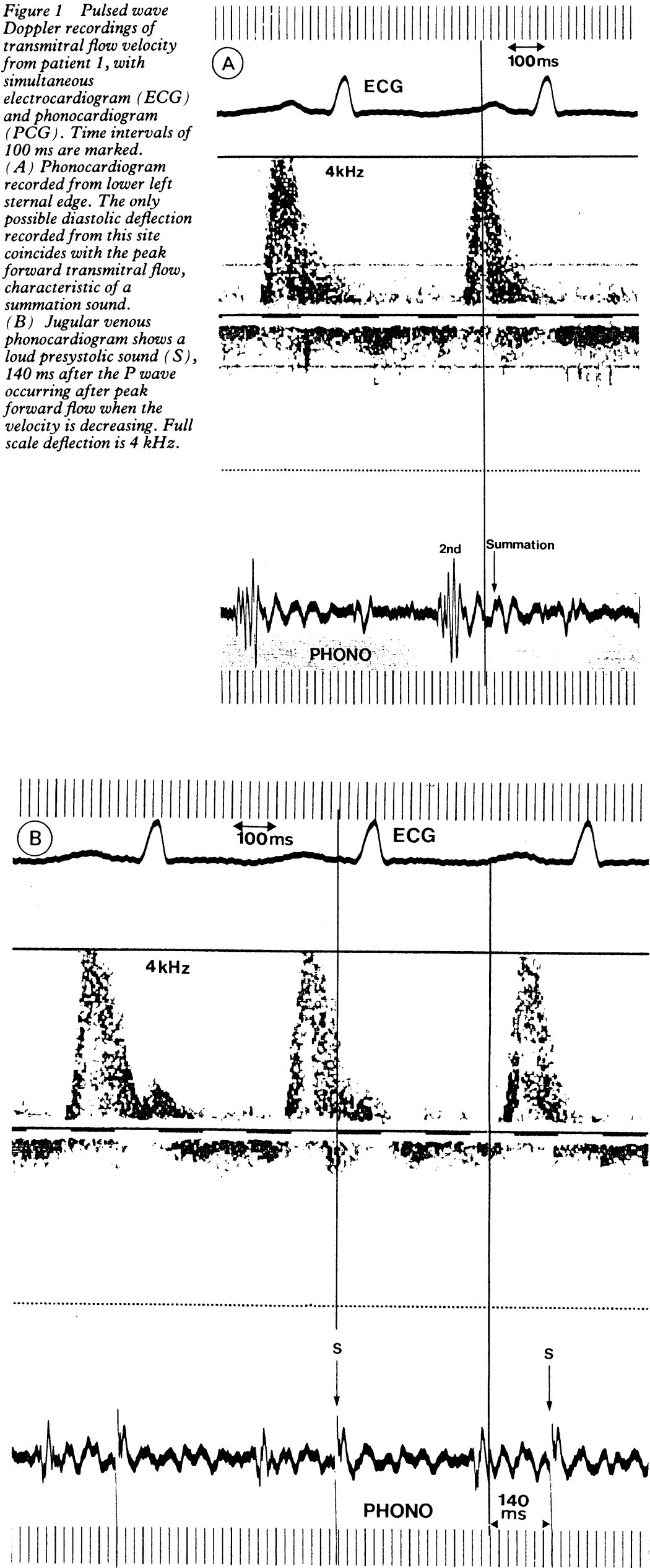

1A). The only possible explanation of this would be a summation sound. No other added sound was recorded from this site, but on the jugular venous phonocardiogram, $140 \mathrm{~ms}$ after the start of the $P$ wave, a second, loud high frequency vibration was present (fig 1B). This sound was localised to an area at the base of the neck and upper right sternal edge. It could not be recorded by placing the microphone lower down the sternal edge or at the apex. The short diastolic filling time, localised nature, and timing in relation to the $P$ wave made it unlikely to be a fourth heart sound, so a detailed Doppler, echo, and phono cardiographic study was performed to identify its origin.

The first and second heart sounds occurred normally in association with mitral and aortic closure identified from the corresponding echograms. Mitral and tricuspid regurgitation were present, limiting the time available for filling of the left ventricle to $220 \mathrm{~ms}$, and the right to $260 \mathrm{~ms}$. On the transmitral Doppler recording a single peak of forward flow was identified and the only possible added sound recordable from the left sternal edge coincided with the peak velocity of left ventricular inflow, characteristic of a summation sound (fig 1A). The sound under study occurred $90 \mathrm{~ms}$ after this and $40 \mathrm{~ms}$ after peak forward tricuspid flow while the velocity of atrioventricular flow on both sides of the heart was steadily decreasing. Premature closure of the tricuspid valve ${ }^{3}$ was excluded by showing normal timing on the echocardiogram of the tricuspid valve, coincident with the first heart sound. As the maximal amplitude of the sound was recorded with the transducer placed beneath the right clavicle, we considered the possibility of the origin of the sound being within the superior vena cava. We therefore recorded pulsed wave Doppler signals from the right supraclavicular fossa directed towards the right atrium (fig 2). A simultaneous jugular venous pulse recording showed that the " $x$ " descent was shallow, but the " $y$ " descent was dominant and deep, indicating predominant early diastolic antegrade flow into the right atrium characteristic of a restrictive filling pattern. Also the apexcardiogram was strongly suggestive of restricted ventricular filling. Diastolic downward flow was recorded from the superior vena cava to the right atrium on Doppler. On the spectral display a rapid halt to forward flow could be seen with subsequent flow reversal (peak forward velocity $0.5 \mathrm{~m} / \mathrm{s}$; acceleration in a retrograde direction $7.7 \mathrm{~m} / \mathrm{s}^{2}=0.79 \mathrm{~g}$ ) occurring before ventricular systole, with retrograde flow from the right atrium towards the superior vena cava. The start of the high-pitched added sound occurred at the time of peak velocity of inflow to the right atrium and coincided with abrupt deceleration.

PATIENT 2

A 29 year old woman with primary pulmonary hypertension was referred for assessment for heart lung transplantation. She had been born a twin by caesarean section. At school she had been breathless on mild exertion and had 

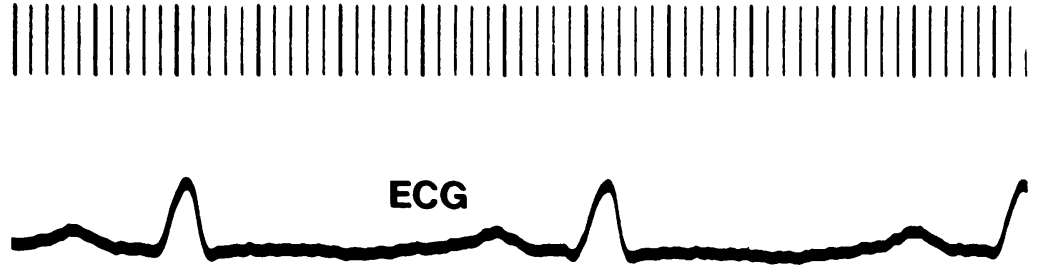

$2 \mathrm{kHz}$

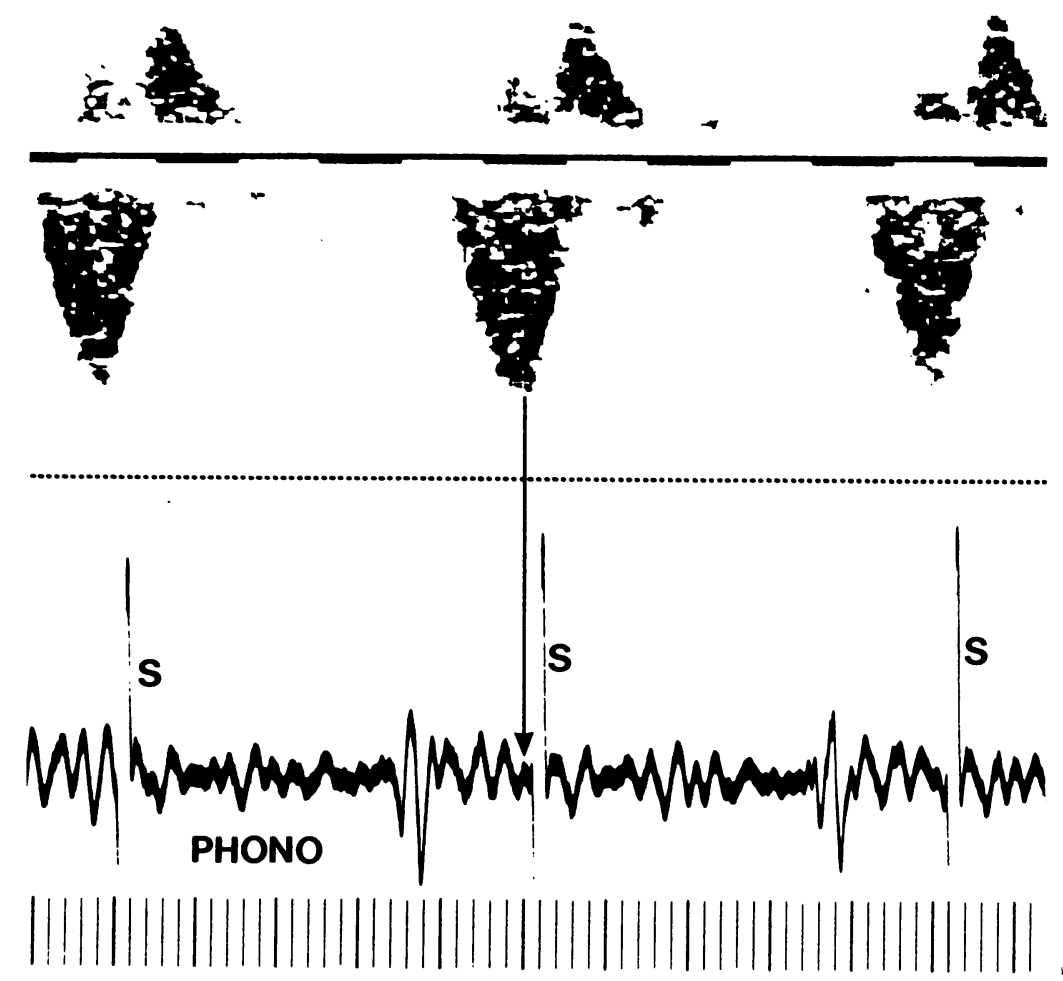

Figure 2 Pulsed wave Doppler flow of the superior vena cava recorded from the right supraclavicular fossa directed towards the right atrium from patient 1. Simultaneous electrocardiogram (ECG) and jugular venous phonocardiogram (PCG) are displayed. Loud presystolic "superior vena cava sound" $(S)$, the start of which coincides with the peak velocity into right atrium at a point of abrupt deceleration. Full scale deflection is
$2 \mathrm{kHz}$. catheterisation, pulmonary artery pressure was recorded as $170 / 110 \mathrm{~mm} \mathrm{Hg}$. A diagnosis of primary pulmonary hypertension with a patent foramen ovale was made.

Echocardiography showed a small left ventricular cavity and a hypertrophied and mildly dilated right ventricle. Tricuspid regurgitation was present with a peak right ventricular atrial gradient of $110 \mathrm{~mm} \mathrm{Hg}$ being recorded. Phonocardiography showed a presystolic sound recorded over the upper right sternal edge $130 \mathrm{~ms}$ after the start of the $P$ wave. The transtricuspid Doppler signal (fig 4A) showed that forward atrioventricular flow had ceased and eliminated the possibility of this being a fourth heart sound. Thus despite electrical and mechanical evidence of atrial activity no atrial transtricuspid flow could be detected, implying a fixed end diastolic right ventricular volume into which no further filling could occur. Superior vena caval flow was examined with Doppler from the supraclavicular fossa (fig 4B). Flow reversal could be detected, with clear retrograde flow from right atrium to superior vena cava. The peak velocity of this retrograde flow $(0.55 \mathrm{~m} / \mathrm{s}$, acceleration, $9.6 \mathrm{~m} / \mathrm{s}^{2}=0.98 \mathrm{~g}$ ) occurred $130 \mathrm{~ms}$ after the beginning of the $P$ wave. The start of the added presystolic sound coincided with the peak retrograde velocity, when the acceleration changed to deceleration.

\section{PATIENT 3}

A 57 year old West Indian woman with chronic pulmonary thromboembolic disease was referred for assessment because of progressive dyspnoea for eighteen months. Her pulse rate was 90 beats per minute and regular and blood pressure was $160 / 100 \mathrm{~mm} \mathrm{Hg}$. The venous pressure was raised with a prominent " $a$ " wave visible. A prominent right ventricular impulse was palpable, a fourth heart sound was audible, and the pulmonary component of the second sound was prominent. Chest $x$ ray film showed enlarged proximal pulmonary arteries and an enlarged cardiac shadow. The electrocardiogram showed right axis deviation, $\mathrm{P}$ pulmonale, and inverted $T$ waves over leads V1-V4. At cardiac catheterisation the following pressures were recorded $(\mathrm{mm} \mathrm{Hg})$ : right atrium, a 17, mean 13; right ventricle $75 / 14$; main pulmonary artery $70 / 30$, mean 45 . Pulmonary angiography showed filling defects in the right main and both lower lobe pulmonary arteries.

Echocardiography showed the right ventricle to be dilated with reversal of septal motion. Prolonged tricuspid regurgitation shortened the right sided filling time to less than $200 \mathrm{~ms}$. A single peak was present on the Doppler recording of forward tricuspid flow, the start of which coincided with a fourth heart sound recorded at the right sternal edge. A louder presystolic sound was recorded over the right jugular veins. Simultaneous jugular phonocardiography and recording of superior vena caval flow on Doppler (fig 5) showed that this presystolic sound coincided with the start of retrograde flow in the superior vena cava after atrial systole (peak retrograde velocity $0.34 \mathrm{~m} / \mathrm{s}$; acceleration $15.4 \mathrm{~m} / \mathrm{s}^{2}=1.57 \mathrm{~g}$ ). apex. The pulmonary component of the second heart sound was accentuated. The electrocardiogram showed right axis deviation and chest $x$ ray film showed enlarged pulmonary arteries with peripheral pruning. At cardiac

avoided strenuous activities. Symptoms procatheterisation at another hospital, a diagnosis of primary pulmonary hypertension had been consideration for heart-lung transplantation. On examination she was centrally cyanosed and giant "a" wave clearly visible (fig 3). Blood pressure was $110 / 70 \mathrm{~mm} \mathrm{Hg}$, the right ventricular impulse was prominent, and on auscultation, a gallop rhythm together with a 
|||||||||||||||||||||||||||||||||||||||||||||||||||||||||||||
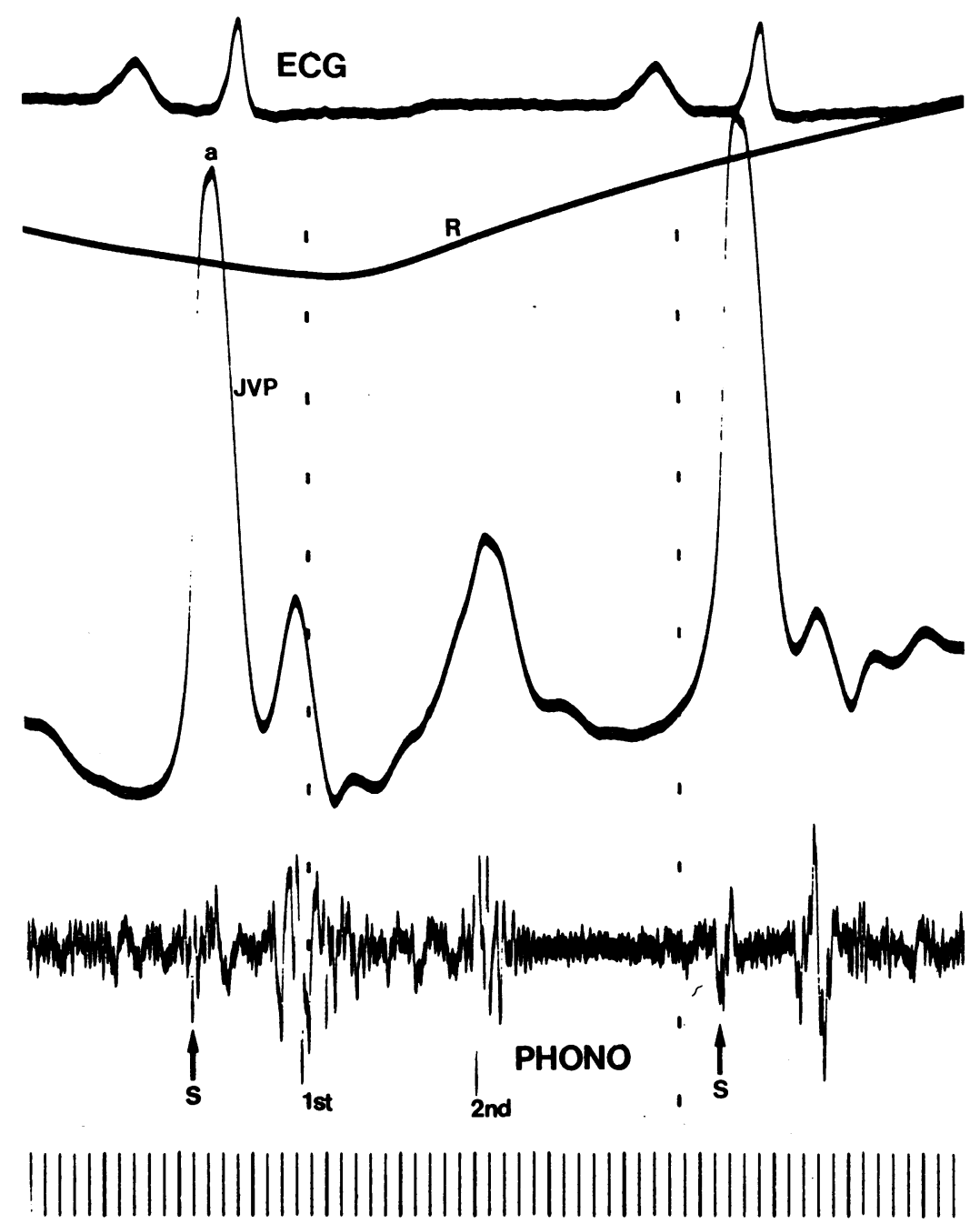

Figure 3 Recording of jugular venous pulse (JVP) from patient 2, with simultaneous electrocardiogram (ECG), phonocardiogram (PCG), and respiratory trace $(R)$, from patient 2. Giant " $a$ " wave coincides with presystolic sound $(S)$. First and second heart sounds are indicated. wall during early diastole. ${ }^{6}$ Fourth sounds usually occur at the beginning of atrioventricular flow after atrial systole, when acceleration of blood starts. ${ }^{2}$ Apart from mitral and tricuspid opening snaps, the only other commonly recognised diastolic sound is a pericardial knock. This is an early diastolic sound heard along the left sternal edge in constrictive pericarditis, and corresponds to the sudden end of ventricular filling and the premature diastolic plateau of the ventricular volume curve. ${ }^{7}$ It occurs simultaneously with the nadir of the " $y$ " descent on the recording of jugular venous pressure. We have already published a case report of a sound during isovolumic relaxation due to intracavity flow. ${ }^{8}$ This sound occurred at the time of peak flow velocity when acceleration had stopped and deceleration had started. Thus the general mechanism of diastolic sound production seems to be related to the dissipation of energy associated with sudden changes in acceleration.

The presystolic sounds in our patients had none of the usual characteristics by which we recognise common diastolic sounds. In case 1 the sound occurred after the peak forward transmitral flow and was not associated with atrial systolic flow into the ventricle. We confirmed that it was not due to premature tricuspid closure $^{3}$ (the normal PR interval made this unlikely). Premature mitral closure has been described in a patient with aortic regurgitation and first degree heart block. ${ }^{9}$ Again, examination of the Doppler trace and mitral echogram confirmed that this could not be the explanation. The other unusual feature was the specific localisation over which the sound could be recorded. This was limited to an area beneath the right clavicle in the parasternal region. The diastolic sound in this patient corresponded in time to a change in acceleration associated with subsequent flow reversal in the Doppler recording from the superior vena cava. Acceleration of blood had ceased and deceleration had started, and blood flow in the superior vena cava subsequently became retrograde. The sudden change of acceleration to deceleration requires the operation of a force and dissipation of energy that appears as mechanical vibrations generating the added sound and may occasionally even be palpable in the venous pulse. The change in acceleration responsible for the added sound in this case is analogous to that responsible for a third heart sound, but comes from different circulatory structures. The question arises why such a change in acceleration and abnormal flow reversal should occur. This patient's apex cardiogram strongly suggested restricted ventricular filling. The patient's previous myocarditis had progressed to cause substantial ventricular impairment and it seems reasonable that such a ventricle could display restrictive properties. Hence, during rapid early diastolic filling a point is reached where atrioventricular blood flow rapidly decelerates yet venous return from the great veins continues. In this situation subsequent retrograde flow into the superior vena cava would occur. Possibly the start of atrial systole 


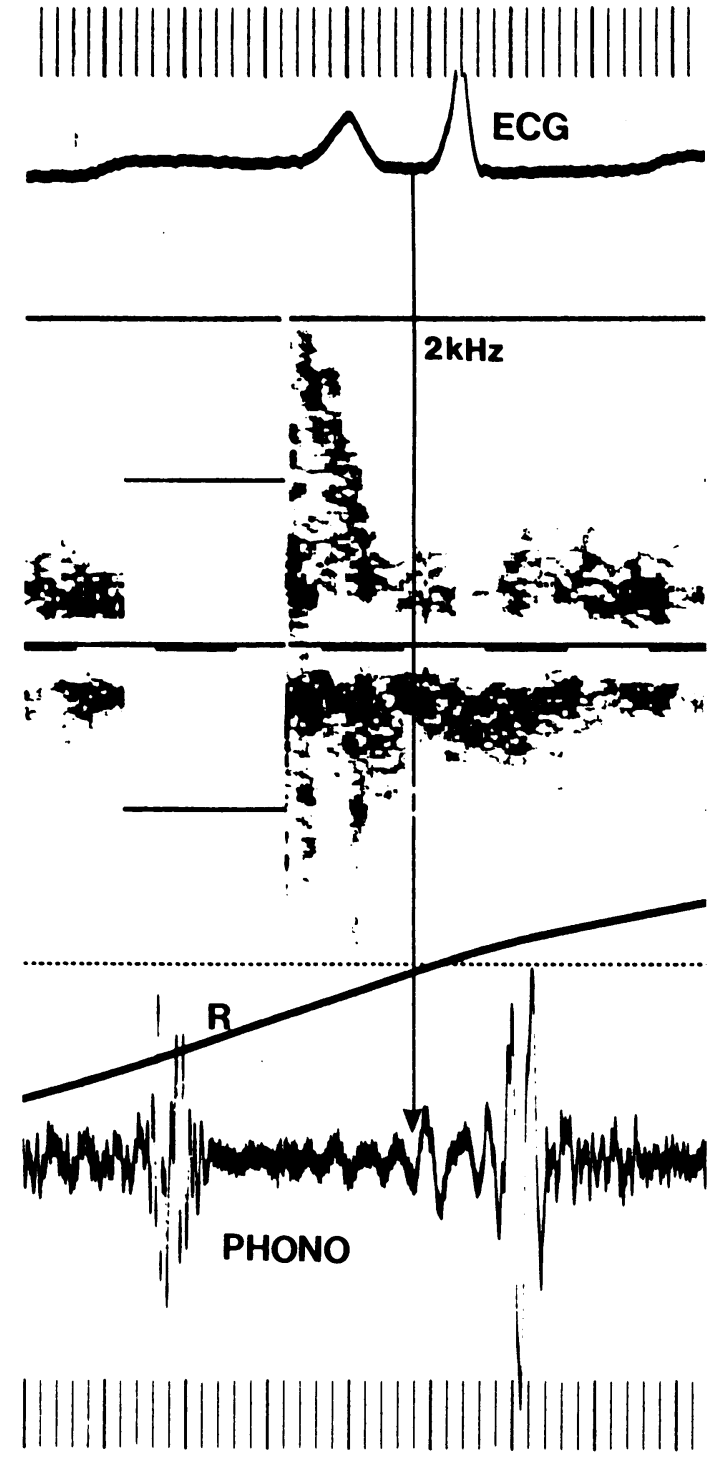

A

Figure 4 Pulsed wave Doppler recording of (A) transtricuspid flow velocity, and (B) superior vena caval flow, from patient 2, with simultaneous electrocardiogram (ECG), phonocardiogram (PCG), and respiratory trace $(R)$. ( $A)$ Phonocardiogram recorded from the upper right sternal edge reveals a presystolic sound (arrowed), occurring after peak forward transtricuspid flow, when flow had ceased.

$(B)$ "Superior vena cava sound" $(S)$ occurs at point of maximal retrograde velocity, associated with an abrupt change in acceleration. Full scale deflection is $2 \mathrm{kHz}$.
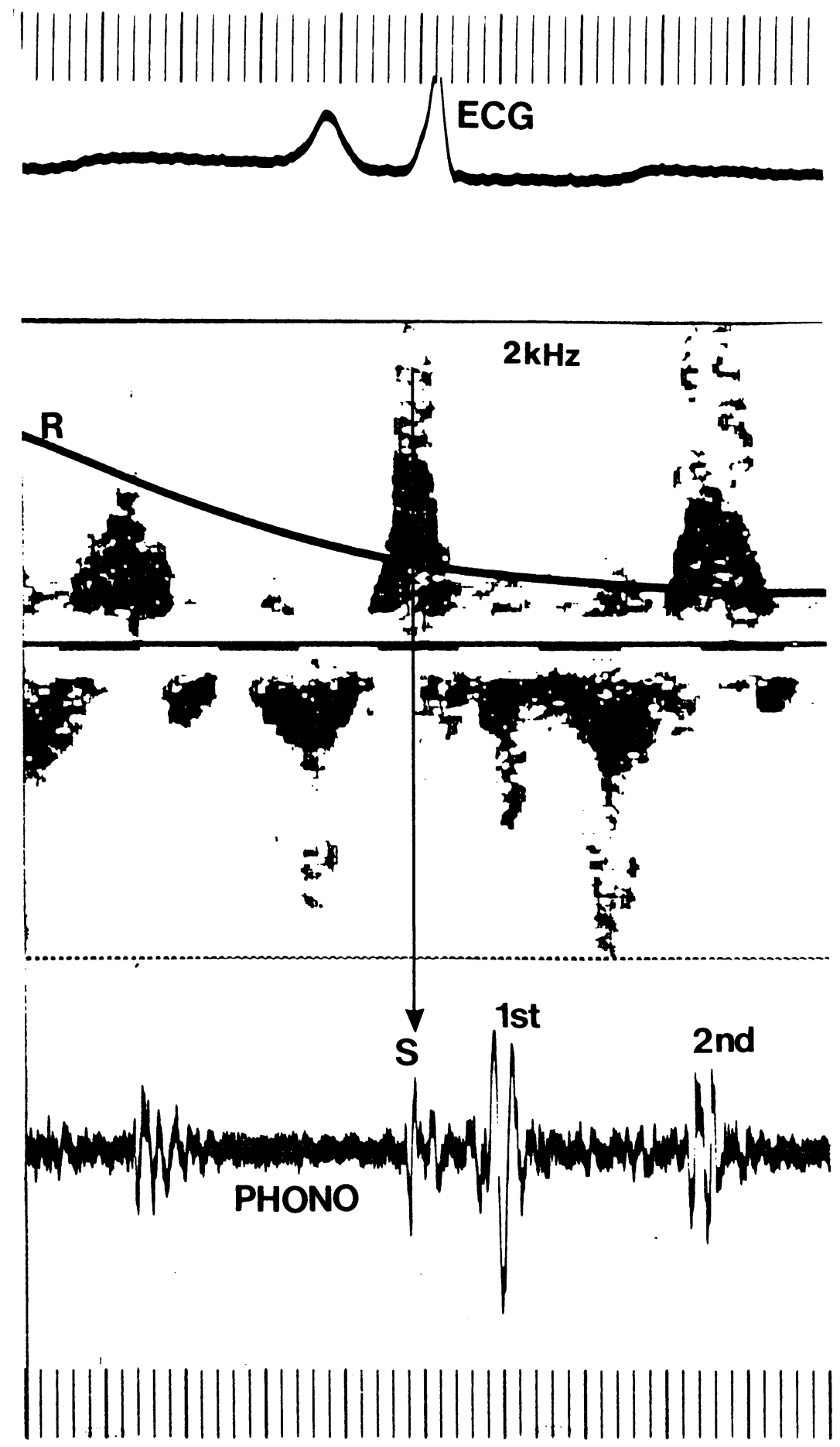

B would increase the pressure gradient underlying this retrograde flow. Normal venous compliance dictates a considerable phase delay between flow and increase in pressure. In this case the flow and pressure changes in the superior vena cava were in phase, indicative of reduction in compliance or increased stiffness of the venous system. This would be expected when the venous pressure itself is greatly raised and provides further support for the venous origin of the sound under discussion. The high-pitched nature of the sound might be a measure of the stiffness of the vein.

In our second case the timing of the added sound was later and occurred $130 \mathrm{~ms}$ after the start of the $P$ wave. At first sight, this has the characteristics of a fourth heart sound, being coincident with the "a" wave. Forward tricuspid flow and hence ventricular filling had stopped at this point, however, making the possibility of atrioventricular origin of this sound unlikely. In this case the sound followed a rapid reversal of flow in the superior vena cava, occurring at the point of peak velocity of flow away from the atrium. The magnitude of the change in acceleration was greatest in the retrograde direction, which may explain the difference in timing from the first case. In both cases 1 and 2, however, the change from acceleration to deceleration implies a change in 

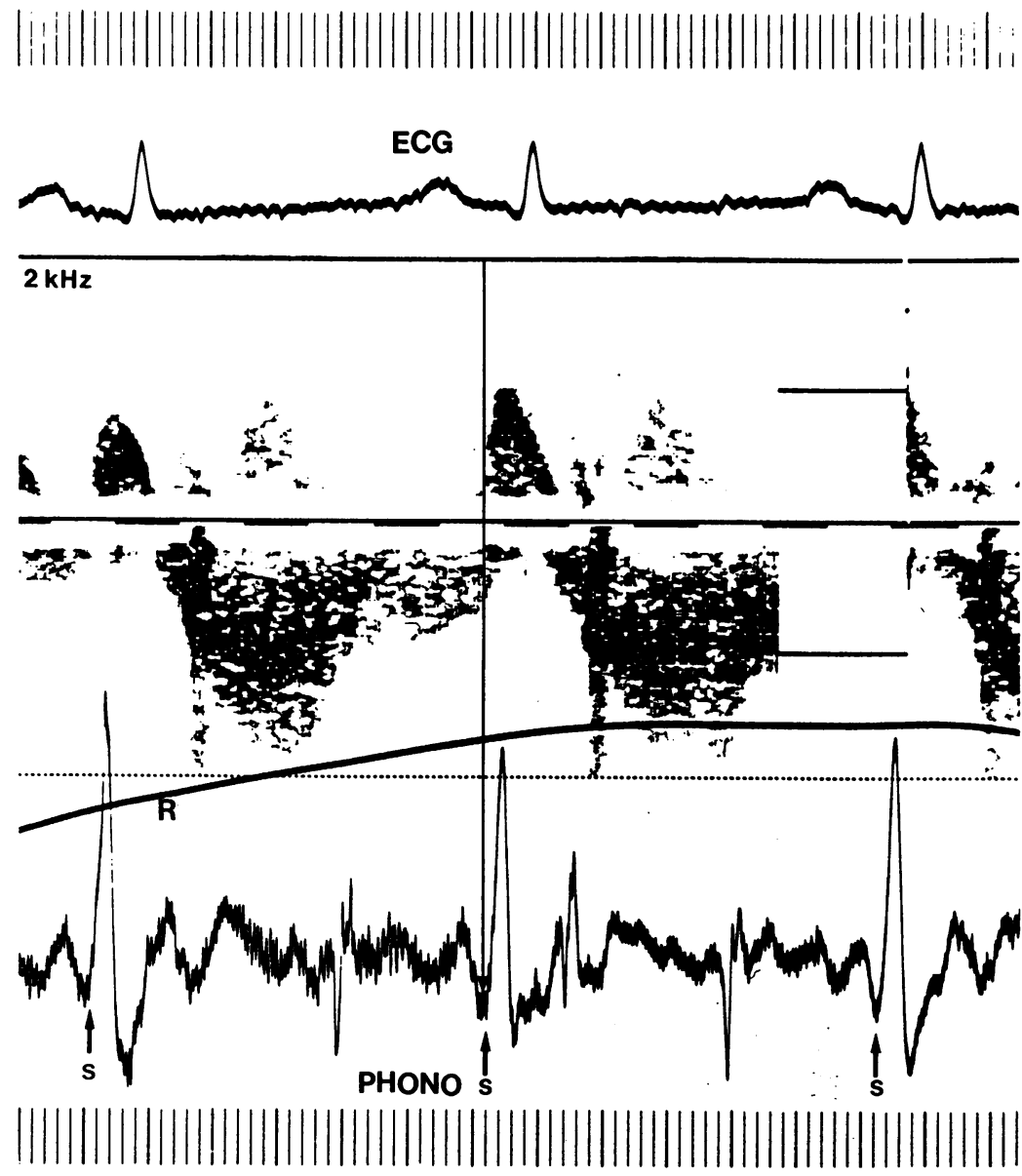

Figure 5 Pulsed wave Doppler recording of superior vena caval flow from patient 3, with simultaneous electrocardiogram (ECG), jugular phonocardiogram (PCG) and respiratory trace $(R)$. The presystolic sound $(S)$ occurs at the start of retrograde flow in the superior vena cava, again associated with a change in acceleration. Full scale deflection is $2 \mathrm{kHz}$. various pathological conditions. In two cases the diagnosis was tricuspid stenosis, and it was postulated that reflux of blood into the great veins set up vibration in the tensed veins so causing a loud sound. This sound was not audible, however, below the clavicle. The postulated mechanism was violent retrograde flow at the height of atrial systole associated with high right ventricular end diastolic pressure, or tricuspid stenosis. We can find no reference to these sounds in publications since 1956, although our experience suggests that they are not uncommon. In our patients we postulate a similar mechanism to that suggested by Dock, and have been able to show the sudden change in acceleration of blood associated with flow reversal. We thus extend Dock's ideas by suggesting that it is the dissipation of energy associated with this sudden acceleration change that generates vibrations within the great veins and hence the sound. In the first two cases, the sound can be shown to occur at a time of peak velocity, when acceleration has ceased. In the first case this occurred at the point of peak forward velocity of flow from the superior vena cava to the right atrium. In the second case the sound coincided with peak reverse velocity from the right atrium to the superior vena cava. In our third case the sound occurred at the start of rapid acceleration of blood in a retrograde fashion, more in keeping with the mechanism of a classical fourth heart sound. Thus this previously described but little recognised class of sound can be shown to have the same basic mechanisms as classical ventricular third and fourth heart sounds.

We believe a suitable name for this added sound is a "superior vena cava sound." Alternative titles such as "fifth sound" may be confusing and "presystolic sound" tells us little about the mechanism producing it. It may be that different classes of this sound will be further defined according to their precise time relations to the flow reversal-for example, "forward flow," "reverse flow," or "acceleration" sounds. Nevertheless, for the time being the term "superior vena cava sound" seems attractive and defines the site of flow reversal.

Phonocardiography has become less popular in recent years. Nevertheless, its use with Doppler and echocardiography makes it possible to describe and explain a class of heart sound, virtually unrecognised since its description by Mackenzie over 80 years ago. Physicians should be alert to the presence of such sounds, as with increased understanding of their genesis, such physical signs may continue to provide valuable information about ventricular physiology.

We thank Dr Martin St John Sutton, Dr Jane Somerville, and Wr thaul Oldershaw for allowing us to discuss their patients in the Dr Paul Oldershaw

S J D B is supported by a British Heart Foundation junior research fellowship. opposite to the flow of blood "11 Eight years later Dock described the recording of loud sounds during atrial systole over the jugular veins of patients with raised right atrial pressure. $^{12}$ Twelve cases were described with 
Br Heart J 1989;61:144-8.

3 Lee $\mathrm{CH}$, Xiao HB, Gibson DG. Presystolic tricuspid valve closure: An alternative mechanism of diastolic sound genesis. Cardiology 1990;77:340-5.

4 Laennec RTH. Traité de l'auscultation médiate. 2nd ed. Paris: Brosson et Chaude, 1826

5 Ozawa Y, Smith D, Craige E. Origin of the third heart sound: I. Studies in dogs. Circulation 1983;67:393-8.

6 Ozawa Y, Smith D, Craige E. Origin of the third hear sound: II. Studies in human subjects. Circulation

7 Tyberg TI, Goodyer AVN, Langou RA. Genesis of a pericardial knock in constrictive pericarditis. Am Cardiol 1980;46:570-5.
8 Lee $\mathrm{CH}$, Gibson DG. Isovolumic relaxation sound: a new class of added heart sound? Br Heart J 1991;65:357-9.

9 Traill TA, Fortuin N. Presystolic mitral closure sound in aortic regurgitation with left ventricular hypertrophy and aortic regurgitation with left ventricular hypertrophy
first degree heart block. Br Heart J 1982;48:78-80.

10 Mackenzie J. Valvular defects (continued). In: Mackenzie J ed. Diseases of the heart. London: Oxford University Press,

11 Groedel FM, Miller M. Studies on the acoustic phenomen over the vessels of the neck in the healthy and diseased heart. Exper Med Surg 1944;11:193-215.

12 Dock W. Loud presystolic sounds over the jugular veins associated with high venous pressure. $\mathrm{Am} \mathrm{J} \mathrm{Med} 1956$ 20:853-9.

\section{Prediction of sudden death in hypertrophic cardiomyopathy}

Abnormalities of heavy chain myosin account for perhaps $50 \%$ of hypertrophic cardiomyopathy (HCM). It now seems that the site and nature of the aminoacid change influence survival. Why should this be? One possibility is that the substitutions interfere to a different degree in myofibrillary organisation within the myocyte. It is probably significant that the substitution leading to a change in charge has the greatest deleterious effect on survival. Abnormal myofibrillary organisation probably leads in turn to misshapen cells and abnormalities in cell to cell organisation producing disarray and an ideal substrate for arrhythmias. Family history remains a cheap way of identifying those with HCM at high risk of sudden premature death but a good case can be made for determining the exact gene abnormality. The understanding of how HCM affects myocyte structure will be further forwarded when the other genes unrelated to heavy chain myosin are discovered. These are exciting times for those interested in HCM.

M J DAVIES

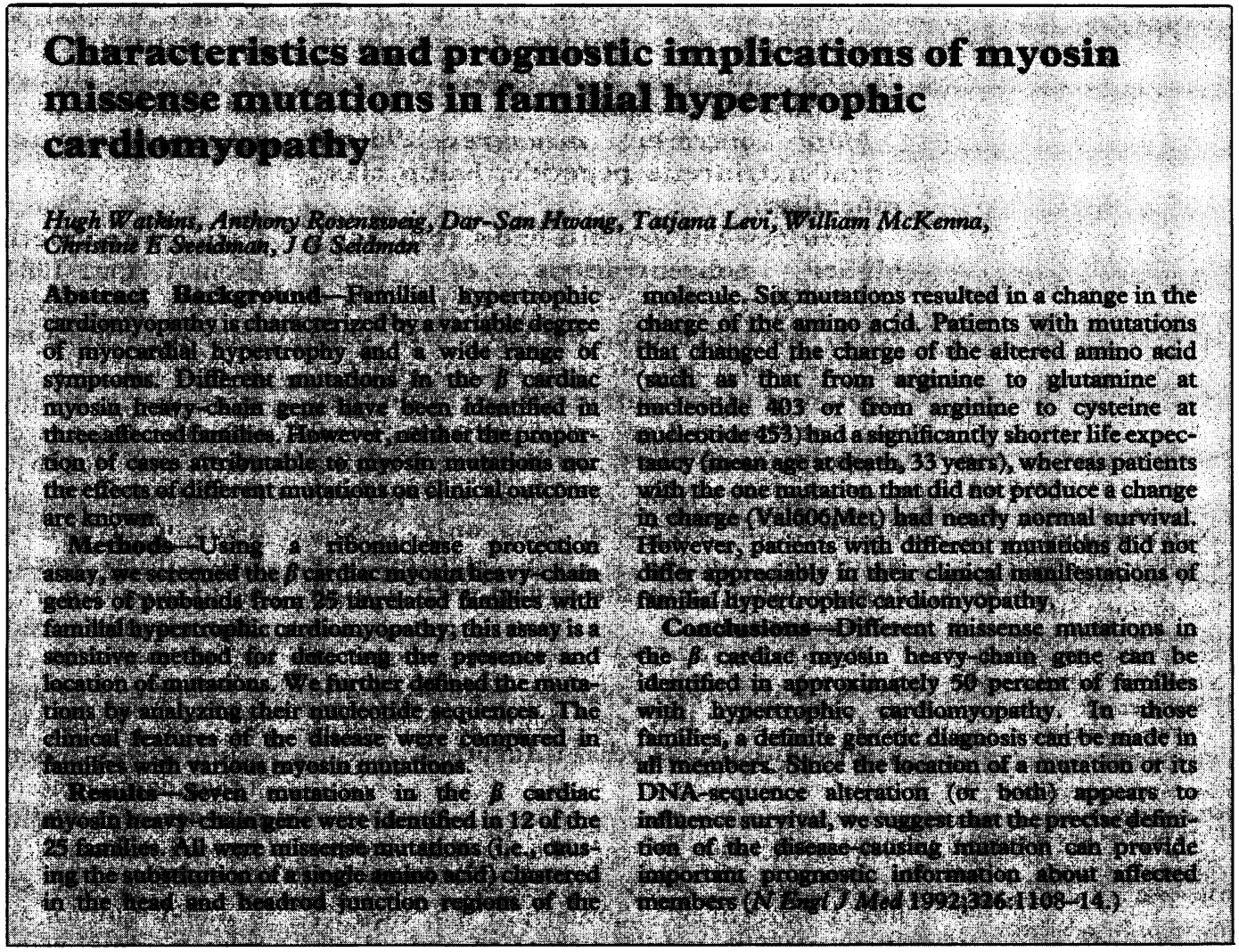

